R. Chapko and B.T. Johansson / Electronic Journal of Boundary Elements, Vol. 7, No. 1, pp. 1-12 (2009)

\title{
An iterative method based on boundary integrals for elliptic Cauchy problems in semi-infinite domains
}

\author{
R. Chapko ${ }^{1}$ and B. T. Johansson ${ }^{2}$ \\ ${ }^{1}$ Faculty of Applied Mathematics and Informatics, Ivan Franko National \\ University of Lviv, 79000 Lviv, Ukraine \\ e-mail: chapko@is.lviv.ua \\ ${ }^{2}$ School of Mathematics, University of Birmingham, Edgbaston, Birmingham \\ B15 2TT, UK \\ e-mail: b.t.johansson@bham.ac.uk
}

\begin{abstract}
In this study, we investigate the problem of reconstruction of a stationary temperature field from given temperature and heat flux on a part of the boundary of a semi-infinite region containing an inclusion. This situation can be modelled as a Cauchy problem for the Laplace operator and it is an ill-posed problem in the sense of Hadamard. We propose and investigate a Landweber-Fridman type iterative method, which preserve the (stationary) heat operator, for the stable reconstruction of the temperature field on the boundary of the inclusion. In each iteration step, mixed boundary value problems for the Laplace operator are solved in the semi-infinite region. Well-posedness of these problems is investigated and convergence of the procedures is discussed. For the numerical implementation of these mixed problems an efficient boundary integral method is proposed which is based on the indirect variant of the boundary integral approach. Using this approach the mixed problems are reduced to integral equations over the (bounded) boundary of the inclusion. Numerical examples are included showing that stable and accurate reconstructions of the temperature field on the boundary of the inclusion can be obtained also in the case of noisy data. These results are compared with those obtained with the alternating iterative method.
\end{abstract}

\section{Introduction}

We consider the following problem:

Given the temperature and heat flux on the boundary of a planar semi-infinite region, reconstruct the (stationary) temperature on the boundary of an inclusion contained in the semi-infinite region.

Semi-infinite regions are common in applied engineering situations, for example, flow and heat conduction in circular cylinders and pipes can give rise to such regions. We start by formulating the above problem mathematically. Assume that 
$D_{1} \subset \mathbb{R}^{2}$ is a semi-infinite region, for example a half-plane, with (unbounded) boundary $\Gamma$, and let $D_{0}$ be a simply connected bounded domain in $\mathbb{R}^{2}$ with boundary $\Gamma_{0} \in C^{2}$, such that $\bar{D}_{0} \subset D_{1}$ (see Fig.1). We define $D:=D_{1} \backslash \bar{D}_{0}$ and $D$ is the unbounded solution domain. The element $\nu$ is the outward unit normal on $\Gamma$. The above stated problem can then be recast into the following Cauchy problem
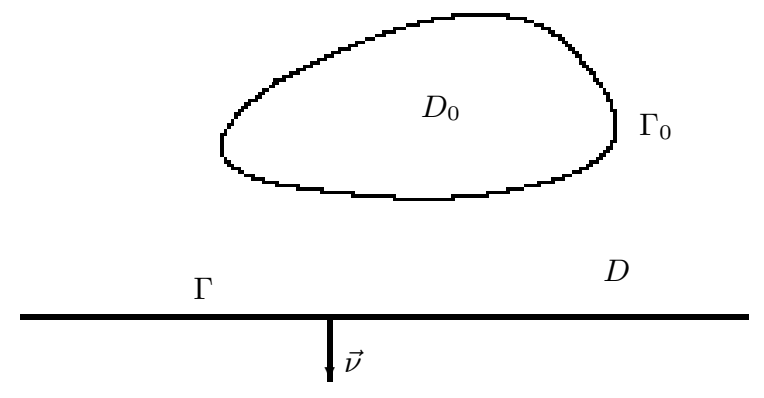

Figure 1: A semi-infinite domain $D$ and its boundaries

of finding a function $u \in C^{2}(D) \cap C^{1}(\bar{D})$ such that

$$
\Delta u=0 \text { in } D
$$

with the boundary conditions

$$
u=f_{1} \quad \text { and } \quad \frac{\partial u}{\partial \nu}=f_{2} \quad \text { on } \quad \Gamma .
$$

We only consider smooth functions $f_{1}$ and $f_{2}$ and it is well-known that (1.1)-(1.2) has at most one solution, see for example [1] and [2]. Due to the importance of the Cauchy problem, many methods have been proposed to reconstruct the solution. Kozlov and Maz'ya [3] proposed an alternating iterative method for solving Cauchy problems for general strongly elliptic and formally self-adjoint systems on bounded domains, which has since been widely used in applied engineering problems. This method was generalized and numerically investigated in Chapko and Johansson [4] for unbounded regions.

In this paper, we propose a Landweber-Fridman procedure that solves mixed problems of the same type throughout the iterations and compare it with the results obtained in [4]. For bounded domains, Landweber-Fridman methods have been applied for Cauchy problems for Helmholtz, heat equation, Laplace equation, linear elasticity and the Stokes system, see, for example, [5], [6], [7], [8] and [9].

The outline is the following. In the next section, we introduce some further notation and formulate the procedure. Also, convergence is discussed, see Theorem 2.1. Then, in Section 3, we briefly outline a boundary integral approach for implementing the mixed problems of the procedure. Numerical investigations are given in Section 4, showing that stable and accurate reconstructions of the temperature field on the boundary of the inclusion can be obtained also in the case of noisy data. 


\section{Formulation and convergence of an iterative procedure for $(1.1)-(1.2)$}

We denote, as usual, by $C^{k}(D)$, where $k$ is a nonnegative integer, the space which consists of all functions having continuous derivatives up to order $k$ on $D$. This is a Banach space under the norm $\|f\|_{C^{k}(D)}=\sup _{0 \leq|\ell| \leq k, x \in D}\left|\partial^{\ell} f(x)\right|$. The space $L^{2}\left(\Gamma_{0}\right)$ is the standard $L^{2}$-space on the (bounded) boundary $\Gamma_{0}$.

We introduce the following mixed boundary value problem:

$$
\begin{gathered}
\Delta u=0 \quad \text { in } D, \\
u=h \quad \text { on } \quad \Gamma_{0}, \quad \frac{\partial u}{\partial \nu}=f_{2} \quad \text { on } \quad \Gamma,
\end{gathered}
$$

and also the following mixed one

$$
\begin{gathered}
\Delta v=0 \quad \text { in } D, \\
v=0 \quad \text { on } \Gamma_{0}, \quad \frac{\partial v}{\partial \nu}=g \text { on } \Gamma .
\end{gathered}
$$

By a solution to (2.1)-(2.2) or (2.3)-(2.4), we mean a classical solution which is uniformly bounded at infinity. Let $f_{1}$ and $f_{2}$ be as in (1.2). The iterative procedure for constructing the solution to (1.1)-(1.2) is:

- Choose an arbitrary function $h_{0}$. Then $u_{0}$ is obtained by solving $(2.1)-(2.2)$ with $u_{0}=h_{0}$ on $\Gamma_{0}$.

- We find $v_{0}$ by solving $(2.3)-(2.4)$ with $\partial v_{0} / \partial \nu=g_{0}$ on $\Gamma$, where $g_{0}=u_{0}-f_{1}$.

- Having constructed $u_{k-1}$ and $v_{k-1}$, the element $u_{k}$ solves $(2.1)-(2.2)$ with $u_{k}=h_{k}$ on $\Gamma_{0}$, where

$$
h_{k}=h_{k-1}-\left.\gamma \frac{\partial v_{k-1}}{\partial \nu}\right|_{\Gamma_{0}}, \quad \gamma>0 .
$$

- Finally, $v_{k}$ is obtained by solving (2.3)-(2.4) with $\partial v_{k} / \partial \nu=g_{k}$ on $\Gamma$, where $g_{k}=u_{k}-f_{1}$.

The procedure continues by iterating in the last two steps.

\subsection{Convergence of the iterative procedure}

It is clear that for sufficiently smooth data (2.1)-(2.2) respectively (2.3)-(2.4), each has a unique bounded classical solution. Thus, we can define the operator $K$ by

$$
K h=\left.u\right|_{\Gamma}
$$


for smooth $h$ where $u$ is a solution to (2.1)-(2.2) with $f_{2}=0$. The operator $K$ is linear. Moreover, using Green's formula it is possible to prove that the adjoint of $K$ is given by

$$
K^{*} g=-\left.\frac{\partial v}{\partial \nu}\right|_{\Gamma_{0}}
$$

for $v$ which solves (2.3)-(2.4). Also, due to the uniqueness of the Cauchy problem, the kernel of $K$ consists of zero only. Further, without loss of generality, we assume that $f_{2}$ is zero. Then the Cauchy problem (1.1)-(1.2) is equivalent to solving the following operator equation

$$
K h=f_{1} .
$$

Using the operator $K$ and its adjoint it is straightforward to check that from the iterative procedure

$$
h_{k}=h_{k-1}-\gamma K^{*}\left(K h_{k-1}-f_{1}\right) .
$$

This is the Landweber-Fridman iteration for solving (2.6); from Engl, Hanke and Neubauer [10, p. 155]

Theorem 2..1 Assume that problem (1.1)-(1.2) has a bounded solution with trace $h$ on the boundary $\Gamma_{0}$ of the inclusion. Let $h_{k}$ be the $k$-th approximate solution on the boundary $\Gamma_{0}$ given by the above procedure. Then, if $\gamma>0$ is chosen sufficiently small, and for any smooth initial data element $h_{0}$

$$
\lim _{k \rightarrow \infty}\left\|h-h_{k}\right\|_{L^{2}\left(\Gamma_{0}\right)}=0 .
$$

Note that there are estimates for the size of $\gamma$ in terms of the norm of the operator $K$, see [10].

We point out that different growth conditions at infinity can be introduced and these can be captured using appropriate weighted spaces, see [11] and [12]. Thus, it is possible to extend this work to such weighted spaces employing results from [7]. We then note that the procedure in Section 2 also works with inexact data since it is well-known that the Landweber-Fridman method with the so-called discrepancy principle is an order optimal regularization method, see for example Engl et al. [10, p. 159].

\section{Numerical solution of the mixed boundary value problem}

In the procedure in the previous section it is needed to numerically solve two mixed boundary value problems in every iteration step. Both are mixed DirichletNeumann problems with different boundary functions. We shall employ a potential boundary layer approach for their. We point out that it is known that the boundary value problems for the Laplace equation in semi-infinite planar domains can be reduced to the case of classical interior problems by using appropriate conformal mappings. However, since we intend to extend this work to three-dimensions and to other differential operators, we do not apply the technique with conformal mappings. Instead, we use a boundary layer approach described in [4]. For the sake of completeness, we give the details for the case of the half-plane. 


\subsection{Boundary integral equation approach}

We search for the solution of the following mixed Dirichlet-Neumann boundary value problem:

$$
\begin{gathered}
\Delta u=0 \quad \text { in } D, \\
u=h \quad \text { on } \Gamma_{0}, \quad \frac{\partial u}{\partial \nu}=f \quad \text { on } \Gamma,
\end{gathered}
$$

which is regular at infinity. The given boundary functions $h$ and $f$ are sufficiently smooth. The special features of the domain $D$ determine our numerical method for the solution of the direct problem (3.1)-(3.2). Since $D$ is an unbounded domain, a very efficient numerical method is the application of the indirect variant of the boundary integral equation approach. Therefore, the single-layer potential approach with a Green's function for the given semi-infinite region $D_{1}$ is used. For a half-plane the corresponding Green's function for the Neumann boundary value condition has the form

$$
N(x, y)=\ln \frac{1}{|x-y|\left|x-y^{*}\right|}, \quad y^{*}:=\left(y_{1},-y_{2}\right) .
$$

Thus, using the single-layer potential approach with the Green's function $N$ for the domain $D_{1}$, we can seek the solution of the mixed problem (3.1)-(3.2) in the form

$$
u(x)=\frac{1}{2 \pi} \int_{\Gamma_{0}} \varphi(y) N(x, y) d s(y)+\frac{1}{2 \pi} \int_{\Gamma} f(y) N(x, y) d s(y)+\alpha, \quad x \in D,
$$

with an unknown density $\varphi$ on $\Gamma_{0}$ and a constant $\alpha$. In order to satisfy the boundedness condition at infinity, the side condition

$$
\frac{1}{2 \pi} \int_{\Gamma_{0}} \varphi(y) d s(y)=0
$$

is imposed. As is evident from the approach (3.3), some additional conditions on the boundary function $f$ must be imposed:

$$
f(x)=O\left(|x|^{-1-\varepsilon}\right), \varepsilon>0,|x| \rightarrow \infty, \quad \int_{-\infty}^{\infty} f\left(x_{1}, 0\right) d x_{1}=0 .
$$

Thus, by the continuity of the single-layer potentials and the properties of the Green's function, the problem (3.1)-(3.2) can be reduced to the pair of integral equations

$$
\left\{\begin{array}{c}
\frac{1}{2 \pi} \int_{\Gamma_{0}} \varphi(y) N(x, y) d s(y)+\alpha=h(x)-\frac{1}{2 \pi} \int_{\Gamma} f(y) N(x, y) d s(y), \quad x \in \Gamma_{0}, \\
\int_{\Gamma_{0}} \varphi(y) d s(y)=0,
\end{array}\right.
$$


to be solved for the density $\varphi$ and the constant $\alpha$.

The well-posedness of the integral equation (3.4) in corresponding Hölder or Sobolev spaces follows from classical results (see [13]).

\subsection{A quadrature method for the integral equation of the first kind}

We assume that the boundary curve $\Gamma_{0}$ is given through a parametric representation

$$
\Gamma_{0}:=\left\{x(t)=\left(x_{1}(t), x_{2}(t)\right): 0 \leq t \leq 2 \pi\right\},
$$

where $x: \mathbb{R} \rightarrow \mathbb{R}^{2}$ is $C^{2}$ and $2 \pi$-periodic with $\left|x^{\prime}(t)\right|>0$ for all $t$. For the unbounded boundary part $\Gamma$ we use the representation $\Gamma:=\left\{x_{\infty}(t)=(t, 0),-\infty<\right.$ $t<\infty\}$.

Using these parameterizations we can transform the system (3.4) into the parametric form

$$
\left\{\begin{array}{c}
\frac{1}{2 \pi} \int_{0}^{2 \pi} \mu(\tau)\left[-\frac{1}{2} \ln \left(\frac{4}{e} \sin ^{2} \frac{t-\tau}{2}\right)+H_{1}(t, \tau)\right] d \tau+\alpha=w(t), \quad t \in[0,2 \pi] \\
\int_{0}^{2 \pi} \mu(\tau) d \tau=0
\end{array}\right.
$$

with the $2 \pi$-periodic smooth kernel $H_{1}$,

$$
w(t):=h(x(t))-\frac{1}{2 \pi} \int_{-\infty}^{\infty} \tilde{f}(\tau) N\left(x(t), x_{\infty}(\tau)\right) d \tau, \quad t \in[0,2 \pi],
$$

the density $\mu(t):=\varphi(x(t))\left|x^{\prime}(t)\right|$ and $\tilde{f}(t):=f\left(x_{\infty}(t)\right)$.

For the full discretization of the integral equation of the first kind (3.5), which has a logarithmic singularity, we apply a quadrature method together with the quadrature rule $[13,14]$ based on trigonometric interpolation. For this purpose, we choose an equidistant mesh by setting $t_{i}:=i \pi / M, i=0, \ldots, 2 M-1, M \in \mathbb{N}$ and use the quadrature rules

$$
\begin{gathered}
\frac{1}{2 \pi} \int_{0}^{2 \pi} f(\tau) d \tau \approx \frac{1}{2 M} \sum_{j=0}^{2 M-1} f\left(t_{j}\right), \\
\frac{1}{2 \pi} \int_{0}^{2 \pi} f(\tau) \ln \left(\frac{4}{e} \sin ^{2} \frac{t-\tau}{2}\right) d \tau \approx \sum_{j=0}^{2 M-1} R_{j}(t) f\left(t_{j}\right)
\end{gathered}
$$

with known weight functions $R_{j}$ (see [13]). 
For the numerical calculation of the integrals in (3.6) we apply the so-called sinc-quadrature rule [15]

$$
\int_{-\infty}^{\infty} f(\tau) d \tau \approx h_{\infty} \sum_{i=-M_{1}}^{M_{1}} f\left(i h_{\infty}\right), \quad M_{1} \in \mathbb{N}, \quad h_{\infty}=\frac{c}{\sqrt{M_{1}}}, c>0 .
$$

After the application of the quadrature method to the integral equations (3.5) and the quadrature rule (3.8) for the computation of the integral in the right-hand side (3.6), we obtain the following system of linear equations

$$
\left\{\begin{array}{c}
\sum_{j=0}^{2 M-1} \tilde{\mu}_{j}\left\{-\frac{1}{2} R_{j}\left(t_{i}\right)+\frac{1}{2 M} H_{1}\left(t_{i}, t_{j}\right)\right\}+\alpha=\tilde{w}\left(t_{i}\right), i=0, \ldots, 2 M-1, \\
\sum_{j=0}^{2 M-1} \tilde{\mu}_{j}=0
\end{array}\right.
$$

to be solved for $\tilde{\mu}_{j} \approx \mu\left(t_{j}\right)$ given the right-hand side

$$
\tilde{w}\left(t_{j}\right):=h\left(x\left(t_{j}\right)\right)-h_{\infty} \sum_{i=-M_{1}}^{M_{1}} \tilde{f}\left(i h_{\infty}\right) N\left(x\left(t_{j}\right), x_{\infty}\left(i h_{\infty}\right)\right), \quad j=0, \ldots, 2 M-1 .
$$

A convergence and error analysis for this numerical scheme is described in [14] in a Hölder space setting and in [13] in a Sobolev space setting. This analysis exhibits the dependence of the convergence rate on the smoothness of the boundary curve $\Gamma_{0}$ and the boundary function $h$.

The normal derivative on $\Gamma_{0}$ and the trace of the solution on $\Gamma$ which are required in the iterative procedure described in Section 2, have the representations according to the properties of the single-layer potential:

$\frac{\partial u}{\partial \nu}(x)=-\frac{1}{2} \varphi(x)+\frac{1}{2 \pi} \int_{\Gamma_{0}} \varphi(y) \frac{\partial N(x, y)}{\partial \nu(x)} d s(y)+\frac{1}{2 \pi} \int_{\Gamma} f(y) \frac{\partial N(x, y)}{\partial \nu(x)} d s(y), x \in \Gamma_{0}$,

and

$$
u(x)=\frac{1}{2 \pi} \int_{\Gamma_{0}} \varphi(y) N(x, y) d s(y)+\frac{1}{2 \pi} \int_{\Gamma} f(y) N(x, y) d s(y)+\alpha, \quad x \in \Gamma .
$$

The corresponding approximations can be obtained using the quadratures (3.7)(3.8).

Note here, that we can reformulate the algorithm of the numerical solution of the Cauchy problem from Section 2 in terms of integral equations. Thus we have the following iteration procedure: 
- Choose an arbitrary function $h_{0}$ and solve by the above quadrature method the integral equation (3.4) with $h=h_{0}$ and $f=f_{2}$.

- Find $u_{0}$ on $\Gamma$ by formula (3.11) and form the boundary function $f=u_{0}-f_{1}$ on $\Gamma$ and choose $h=0$ on $\Gamma_{0}$. Again solve the integral equation (3.4) with these boundary functions and find $\partial v_{0} / \partial \nu$ on $\Gamma_{0}$ by formula (3.10).

- Having constructed $u_{k-1}$ on $\Gamma$ and $\partial v_{k-1} / \partial \nu$ on $\Gamma_{0}$, the next approximation is calculated as

$$
h_{k}=h_{k-1}-\left.\gamma \frac{\partial v_{k-1}}{\partial \nu}\right|_{\Gamma_{0}}, \quad \gamma>0 .
$$

- To find the function $u_{k}$ on $\Gamma$ solve the equation (3.4) with $h=h_{k}$ and $f=f_{2}$. Finally, $\partial v_{k} / \partial \nu$ on $\Gamma_{0}$ is obtained by solving the integral equation (3.4) with $f=u_{k}-f_{1}$ on $\Gamma$ and $h=0$ on $\Gamma_{0}$.

\section{Numerical experiments}

We shall investigate the proposed method using both exact and noisy data. Firstly we would like to remark, that we have used the synthetic Cauchy data in all our numerical experiments. These Cauchy data are constructed in the following way. We solve the mixed Dirichlet-Neumann problem with boundary value conditions $u=h$ on $\Gamma_{0}$ and $\partial u / \partial \nu=f_{2}$ on $\Gamma$ for given boundary function $h$ and $f_{2}$ using the above described boundary integral equation method. Then we find the approximation of the trace of the solution on $\Gamma$ according to (3.11) and use it as the boundary function $f_{1}$. To avoid an inverse crime we solve the corresponding integral equations on a finer mesh. Secondly, in the case of noisy data, random pointwise errors have been added to the values of $u$ on $\Gamma$, with the percentage given in terms of the $L^{2}$-norm.

Ex. 1. We consider the ellipse shaped inclusion (see Fig.2a)

$$
\Gamma_{0}=\{x(t)=(1.5 \cos t, \sin t+1.5), t \in[0,2 \pi]\} .
$$

The synthetic Cauchy data are found as described in the beginning of this section (taking due care to avoid an inverse crime) with

$$
h(t)=\sin 2 t \quad \text { on } \Gamma_{0} \quad \text { and } \quad f_{2}\left(x_{1}, x_{2}\right)=0 \quad \text { on } \Gamma .
$$

Results for the reconstruction of the boundary function on $\Gamma_{0}$ by the LandweberFridman method are presented in Fig. 3 (the dashed line corresponds to the exact solution $h$ ). The corresponding $L^{2}$-error is reflected in Fig. 4. For the computations of the $L^{2}$-norms we used the trapezoidal rule. The following discretization parameters were chosen: $M=64$ and $M_{1}=1000$ and the initial guess $h_{0}=0$ on $\Gamma_{0}$ and $\gamma=1.5$. Moreover, the noise level is $5 \%$. Note that in the case of noisy data we illustrate the result corresponding to the minimum $L^{2}$-error.

Ex. 2. Now we consider the case when the boundary curve $\Gamma_{0}$ is the peanut shaped curve

$$
\Gamma_{0}=\{x(t)=(r(t) \cos t, r(t) \sin t+1.5), t \in[0,2 \pi]\}
$$




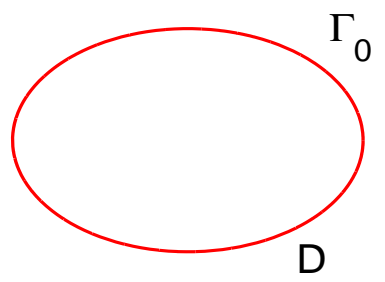

a) The ellipse shaped inclusion

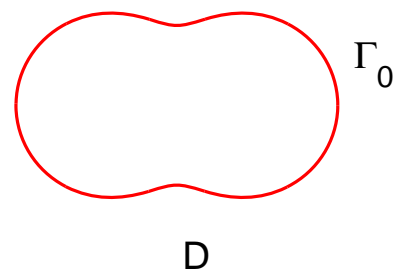

$\Gamma$

b) The peanut shaped inclusion

Figure 2: Solutions domains in the numerical examples

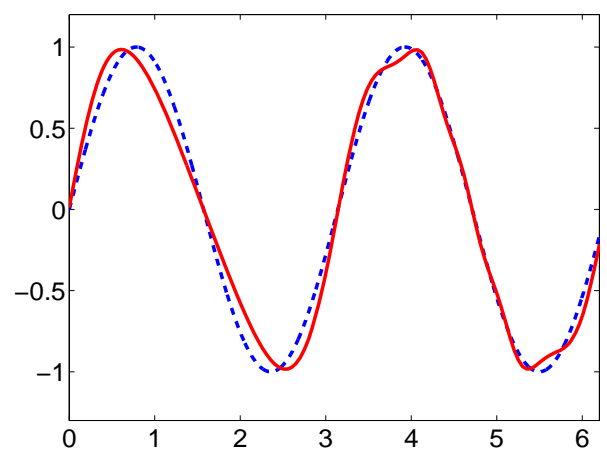

a) Exact data, $k^{*}=2000$

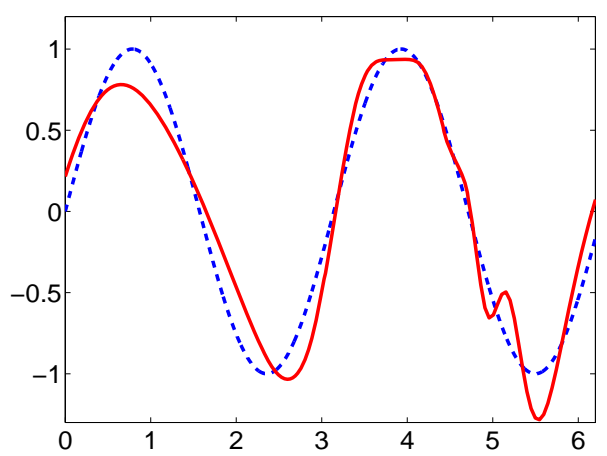

b) $5 \%$ noise, $k^{*}=806$

Figure 3: Reconstruction of the boundary function on the ellipse

with the radial function $r(t)=\sqrt{\cos ^{2} t+0.25 \sin ^{2} t}$ (see Fig.2b). The synthetic Cauchy data are found as described in the beginning of this section with

$h\left(x_{1}, x_{2}\right)=x_{1}-0.1\left(x_{2}-1.5\right) \quad$ on $\Gamma_{0} \quad$ and $\quad f_{2}\left(x_{1}, x_{2}\right)=x_{1} \exp \left(-x_{1}^{2}\right) \quad$ on $\Gamma$.

Results for the reconstruction of the solution on $\Gamma_{0}$ by the Landweber-Fridman method with $\gamma=1$ and the other parameters as in the previous example are presented in Fig. 5 and $L^{2}$ - errors in Fig. 6.

\section{Conclusion}

Based on our numerical experiments we come to the following conclusion:

- The Landweber-Fridman procedure gives an acceptable reconstruction of the temperature on the boundary of the inclusion both for exact and noisy data. We note that the use of the discrepancy principle as a stopping rule, tended to terminate the iterations too early. 


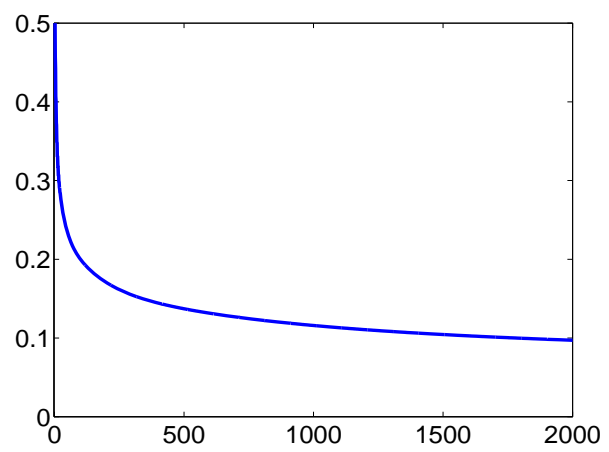

a) Exact data

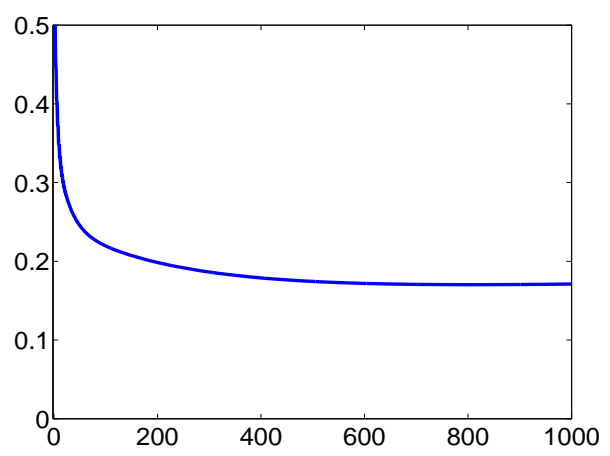

b) Noisy data

Figure 4: $L^{2}$ - errors for the ellipse inclusion

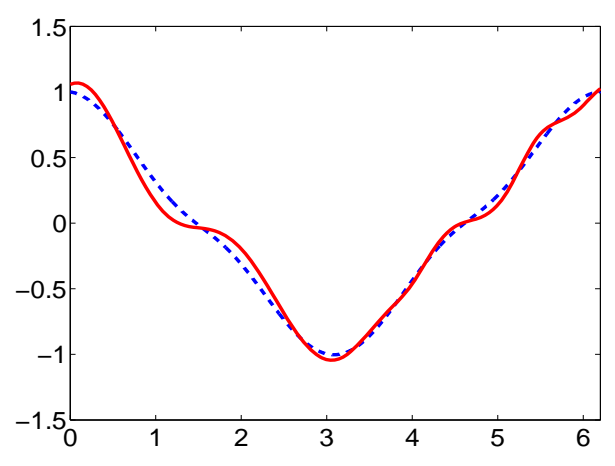

a) Exact data, $k^{*}=1000$

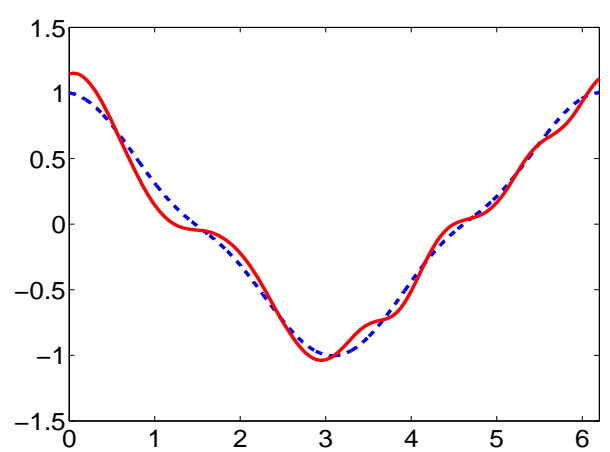

b) $5 \%$ noise, $k^{*}=244$

Figure 5: Reconstruction of the boundary function on the peanut

- The alternating method proposed in [4], which involves the solution of two different mixed problems in every iteration step (each of them solved by the above boundary integral equation method), is more preferable as compared with the Landweber-Fridman iterations. For example, after only 300 iteration steps with the alternating method the reconstruction is more accurate than the reconstruction obtained with the Landweber-Fridman procedure after 1000 steps.

Similar comparisons but for bounded domains are reported in [16].

\section{References}

[1] A.-P. Calderón Uniqueness in the Cauchy problem for partial differential equations, Amer. J. Math. 80, 16-36 (1958). 


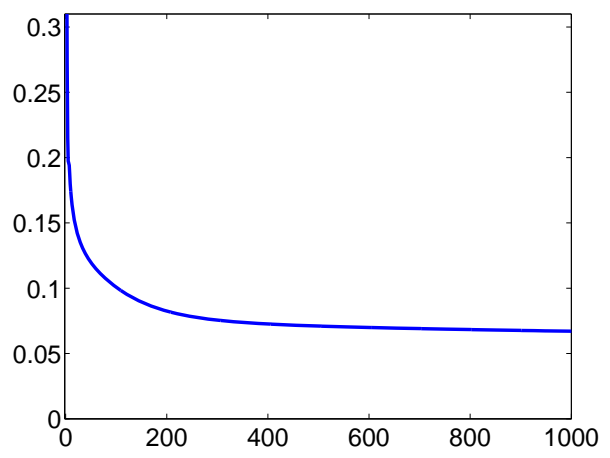

a) Exact data

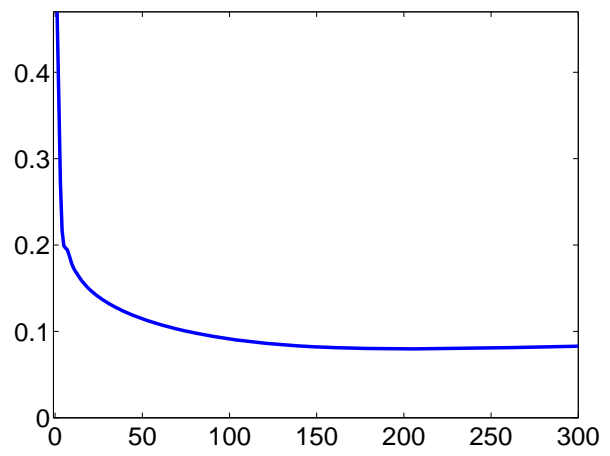

b) Noisy data

Figure 6: $L^{2}$ - errors for the peanut inclusion

[2] T. Carleman Sur un problème d'unicité pur les systèmes d'équations aux partielles à deux variables indépendantes, (French) Ark. Mat., Astr. Fys. 26, 1-9 (1939).

[3] V. A. Kozlov and V. G. Maz'ya On iterative procedures for solving ill-posed boundary value problems that preserve differential equations, Algebra $i$ Analiz 1 (1989), 144-170. English transl.: Leningrad Math. J. 1, 1207-1228 (1990).

[4] R. Chapko and B. T. Johansson An alternating boundary integral based method for a Cauchy problem for Laplace equation in semi-infinite regions, Inverse Problems and Imaging 3, 317-333 (2008).

[5] L. Marin, L. Elliott, P. J. Heggs, D. B. Ingham, D. Lesnic and X. Wen BEM solution for the Cauchy problem associated with Helmholtz-type equations by the Landweber method, Engingn. Anal. Boundary Elements 28, 1025-1034, (2004).

[6] G. Bastay, V. A. Kozlov, and B. O. Turesson Iterative methods for an inverse heat conduction problem, J. Inverse Ill-posed Probl. 9 375-388 (2001).

[7] T. Johansson An iterative procedure for solving a Cauchy problem for second order elliptic equations, Math. Nachr. 272, 46-54 (2004).

[8] L. Marin and D. Lesnic Boundary element-Landweber method for the Cauchy problem in linear elasticity, IMA J. Appl. Math. 70, 323-340 (2005).

[9] T. Johansson and D. Lesnic An iterative method for the reconstruction of a stationary flow, Numer. Methods Partial Differential Equations 23 998-1017 (2007)

[10] H. W. Engl, M. Hanke and A. Neubauer Regularization of Inverse Problems, Kluwer Academic Publishers Group, Dordrecht, (1996). 
R. Chapko and B.T. Johansson / Electronic Journal of Boundary Elements, Vol. 7, No. 1, pp. 1-12 (2009)

[11] C. Amrouche and S. Necasová Laplace equation in the half-space with a nonhomogeneous Dirichlet boundary condition, Math. Bohem. 126 265-274 (2001).

[12] C. Amrouche The Neumann problem in the half-space, C. R. Math. Acad. Sci. Paris 335, 151-156 (2002).

[13] R. Kress Linear Integral Equations, 2nd. ed. Springer-Verlag, Heidelberg (1999).

[14] R. Chapko and R. Kress On a quadrature method for a logarithmic integral equation of the first kind. In Agarwal, ed.: World Scientific Series in Applicable Analysis.- Vol. 2. Contributions in Numerical Mathematics.-World Scientific, Singapore. 127-140 (1993).

[15] F. Stenger Numerical Methods Based on Sinc and Analytic Functions. Springer-Verlag, Heidelberg (1993).

[16] J. Baumeister and A. Leitão On iterative methods for solving ill-posed problems modeled by partial differential equations, J. Inverse Ill-Posed Probl. 9, 13-29 (2001). 